\title{
Early Computed Tomography Findings of Novel Coronavirus Disease 2019 (COVID-19) Pneumonia
}

\author{
Pinar Diydem Yilmaz $(\mathbb{D})^{1,}{ }^{*}$, Cengiz Kadiyoran $\mathbb{B}^{1}{ }^{1}$, Suleyman Bakdik $\mathbb{B}^{1}{ }^{1}$, Necdet Poyraz $(\mathbb{B})^{1}$ and Hulya \\ Vatansev (iD) ${ }^{2}$ \\ ${ }^{1}$ Radiology Department, Meram Faculty of Medicine, Necmettin Erbakan University, Konya, Turkey \\ ${ }^{2}$ Pulmonary Diseases Department, Meram Faculty of Medicine, Necmettin Erbakan University, Konya, Turkey \\ "Corresponding author: Radiology Department, Meram Faculty of Medicine, Necmettin Erbakan University, Konya, Turkey. Email: pinardidemyilmaz@hotmail.com
}

Received 2020 June 25; Accepted 2020 June 28.

\begin{abstract}
Background: Novel coronavirus disease(COVID-19) pneumonia is caused by severe acute respiratory syndrome coronavirus 2 (SARS$\mathrm{CoV}-2$ ), which is highly contagious and threatens human health. Chest computed tomography (CT) is an accurate imaging modality for diagnosis and follow-up.

Objectives: To investigate early CT features of COVID-19 pneumonia.

Methods: Retrospective study. Sixty-three patients with COVID-19 pneumonia (28 men, 35 women; age range, 21 - 85 years; mean, 59 years) confirmed by reverse transcription-polymerase chain reaction (RT-PCR) were enrolled in this retrospective study and CT findings were evaluated. Patients were classified into three groups for estimating COVID-19 pneumonia, according to CT features.

Results: Nineteen (30\%) patients classified as out of COVID-19 pneumonia had normal CT findings. Twenty-eight (44\%) patients, classified as highly suggestive for COVID-19 pneumonia, had typical findings for COVID-19 pneumonia. In highly suggestive for COVID-19 pneumonia group most common CT features were peripheral (64\%), multilobar (96\%), patchy, and round (54\%) ground-glass opacity (GGO) (57\%). Air bronchogram (93\%), crazy-paving patterns (79\%), and vascular thickening (61\%) were the most common special findings. We only detected these rare findings such as halo sign, reverse halo sign, lymphadenopathy, pleural and pericardial effusion, pleural thickening, and fibrotic lines in patients with COVID-19 with chronic diseases.

Conclusions: The early CT findings are peripheral, multilobar, patchy, and round ground glass opacities accompanied by vascular thickening, crazy-paving pattern, or air bronchogram sign. Rare findings are only seen in the presence of concomitant diseases.
\end{abstract}

Keywords: Pneumonia, Computed Tomography (CT), COVID-19, SARS-CoV-2, Coronavirus Disease

\section{Background}

Novel coronavirus disease 2019 (COVID-19), a highly contagious disease caused by severe acute respiratory syndrome coronavirus 2 (SARS-CoV-2), was first reported in Wuhan, China, then spread to worldwide (1). While many countries initially could not predict that the disease would spread so rapidly, the world health organization (WHO) soon identified the virus and declared a pandemic after a quick time after its rapid contamination worldwide (2). Following the findings of upper respiratory tract infection and pneumonia, the entire world faced serious infections resulting in death due to acute respiratory distress. Although it would be limited in China, the mysterious virus, which spread to neighboring countries in a brief time, even showed trans-oceanic spread.

Besides antigen tests and antibody tests, polymerase chain reaction (PCR) plays an important role in COVID19 diagnosis. In the diagnosis of COVID-19, getting viral nucleic acid by real-time PCR (RT-PCR) is considered the standard gold diagnosis (3). However, PCR test positivity in COVID-19 patients is approximately 60\% - 65\%. Besides PCR, additional tests can need to diagnose COVID-19 in patients with one or more of symptoms such as fever, dry cough, shortness of breath, respiratory distress. Lymphocyte count, lactate dehydrogenase, ferritin, and d-dimer values can be useful in both COVID-19 diagnosis and followup its prognosis. The PCR test was negative in many symptomatic and asymptomatic patients with COVID-19 pneumonia. However, these patients were detected early by computed tomography (CT). The PCR test was found to be positive when these patients were screened for the 2 nd or 3rd time. CT sensitivity is $98 \%$ in these patients $(4,5)$, CT 
frequently accomplished early diagnosis of COVID-19.

\section{Objectives}

In this study, we aimed to investigate the CT findings of patients with COVID-19 pneumonia and positive RT-PCR results.

\section{Methods}

We obtained ethics approval for this study from the local Ethics Committee in April 2020. Sixty-three patients with the diagnosis of COVID-19 confirmed by the laboratory by RT-PCR were included in the study. Two experienced thoracic radiologists examined initial CT images of patients with typical clinical symptoms such as fever, dry cough, fatigue, muscle pain, and chest trouble.

All chest CT examinations were performed with Somatom Drive (Siemens Healthineers) scanner. Unenhanced CT scans were obtained from bilateral apex to base for all patients. Scanning parameters were: tube voltage, $120 \mathrm{kV}$; tube current-time product, 50 - $100 \mathrm{mAs}$; pitch, 0.6; matrix, $512 \times 512$; slice thickness, $3 \mathrm{~mm}$, reconstructed slice thickness, $1.5 \mathrm{~mm}$. Images were viewed on Syngo Via Workstation (Siemens Healthineers) in lung window settings (width: 1000 - $1500 \mathrm{HU}$, level:700 to -550 HU) and mediastinal window settings (width: 300 - $350 \mathrm{HU}$, level: 30 - $40 \mathrm{HU}$ ).

We classified the lung changes as follows;

Distribution (peripheral, central, or peripheral and central), the number of affected lobes (one lobe or multilobar), the shapes of lesions (patch, round, or patchy and round), the appearance of lesions (ground-glass opacity [GGO], consolidation, or GGO and consolidation).

We examined specific findings (crazy-paving pattern, air bronchogram, vascular thickening, halo sign, reverse halo sign, and fibrotic lines), and other findings (lymphadenopathy [LAP], pleural thickening, pleural effusion, and pericardial effusion).

Images with typical findings for COVID-19 pneumonia, according to the Radiological Society of North America Expert Consensus, March 2020 (6), were classified as highly suggestive for COVID-19 pneumonia. Images with nonspecific imaging features of COVID-19 pneumonia were classified as suspicious for COVID-19 pneumonia, and CT findings of patients without COVID-19 pneumonia were recorded as normal.

\subsection{Statistical Analysis}

Statistical analysis was performed with SPSS Version 20.0. Whether the data showed, a homogeneous distribution was evaluated with the Kolmogorov-Smirnov test. Categorical data were evaluated by chi-square and Fisher's exact test. Homogeneously distributed data were evaluated by student $t$-test and nonhomogeneous data were evaluated by the Mann-Whitney U-test. A P value $<0.05$ was considered significant.

\section{Results}

Sixty-three patients with laboratory-confirmed for COVID-19 pneumonia (28 men, 35 women; age range, 21 - 85 years; mean, 59 years) were included in the study. Classification of estimating for COVID-19 pneumonia according to CT findings is shown in Table 1. Nineteen (30\%) patients, classified as out of COVID-19 pneumonia, had normal CT findings. Twenty-eight (44\%) patients, classified as highly suggestive for COVID-19 pneumonia, had typical findings for COVID-19 pneumonia. When the patients were divided into three groups as $<40$ years old, 40 - 60 years old and $<60$ years old, the number of patients highly suggestive COVID-19 pneumonia in the 40 - 60 age group was higher than the other groups $(\mathrm{P}=0.022)$. Suspicious and highly suggestive COVID-19 pneumonia numbers were similar in patients with hypertension and diabetes. All results are summarized in Table 1.

Thirty-two (73\%) patients had lesions in peripheral distribution (Figure1), and 31 (70\%) patients had multilobar involvement (Table 2). The most common lung parenchyma changes on CT were patchy and round (39\%) shaped, GGO (61\%) lesions (Figures 2 and 3). Specific and other findings in the initial CT of patients with COVID-19 pneumonia are shown in Table 2. Twenty-seven (61\%) patients had air bronchogram signs (Figure 4), 22 (50\%) had the crazy-paving pattern (Figure 5). Vascular thickening (Figures 6 and 7) was found in 17 (39\%) patients. Halo sign, reverse halo sign, LAP(Figure 8), and pleural effusion was seen only by one patient (2\%). Pericardial effusion and pleural thickening were visualized in $2(5 \%)$ patients. Four $(9 \%)$ patients had fibrotic lines.

When highly suggestive for the COVID-19 pneumonia group was evaluated, the most common CT features (Table 3) were peripheral (64\%), multilobar (96\%), patchy, and round (54\%) GGO (57\%). Air bronchogram (93\%), crazypaving patterns (79\%), and vascular thickening (61\%) were the most common special findings. 


\begin{tabular}{|c|c|c|c|}
\hline & No pneumonia $(\mathrm{N}=19)$ & Suspicious COVID-19 Pneumonia $(\mathrm{N}=16)$ & Highly Suggestive COVID-19 Pneumonia $(\mathrm{N}=28)$ \\
\hline \multicolumn{4}{|l|}{ Age, $y^{\text {b }}$} \\
\hline $20-40(n=6)$ & $3(15.8)$ & $2(12.5)$ & $1(3.6)$ \\
\hline $40-60(n=26)$ & $4(21.0)$ & $4(25.0)$ & $18(64.3)$ \\
\hline$>60(\mathrm{n}=31)$ & $12(63.2)$ & $10(62.5)$ & $9(32.1)$ \\
\hline \multicolumn{4}{|l|}{ Gender } \\
\hline Male $(n=28)$ & $8(42.1)$ & $6(37.5)$ & $14(50.0)$ \\
\hline Female $(\mathrm{n}=35)$ & $11(57.9)$ & $10(62.5)$ & $14(50.0)$ \\
\hline Hypertension & $5(26.3)$ & $4(25.0)$ & $11(39.3)$ \\
\hline Coronary artery disease & $3(15.8)$ & $1(6.3)$ & $3(10.7)$ \\
\hline Diabetes mellitus & $3(15.8)$ & $3(18.8)$ & $14(22.2)$ \\
\hline Hypothyroidism & $1(5.3)$ & 0 & $2(7.1)$ \\
\hline Behçet's disease & 0 & $1(6.3)$ & $1(3.6)$ \\
\hline Chronic renal failure & 0 & 0 & $1(3.6)$ \\
\hline \multicolumn{4}{|l|}{$\begin{array}{l}{ }^{a} \text { Values are expressed as No. (\%). } \\
{ }^{b} P=0.022 .\end{array}$} \\
\hline Findings & All Pr & nonia Groups $(N=44)$ & Suggestive COVID-19 Pneumonia $(\mathrm{N}=28)$ \\
\hline \multicolumn{4}{|l|}{ Distribution of lesions } \\
\hline Peripheral & & $32(73)$ & $18(64)$ \\
\hline Central & & $2(4)$ & $1(4)$ \\
\hline Peripheral and central & & $10(23)$ & $9(32)$ \\
\hline \multicolumn{4}{|l|}{ Number of lobes } \\
\hline One lobe & & $13(30)$ & $1(4)$ \\
\hline Multilobes & & $31(70)$ & $27(96)$ \\
\hline \multicolumn{4}{|l|}{ Shapes of lesions } \\
\hline Patchy & & $11(25)$ & $11(39)$ \\
\hline Round & & $16(36)$ & $2(7)$ \\
\hline Patchy and round & & $17(39)$ & $15(54)$ \\
\hline \multicolumn{4}{|l|}{ Appearance of lesions } \\
\hline GGO & & $27(61)$ & $16(57)$ \\
\hline Consolidation & & $4(9)$ & $1(4)$ \\
\hline GGO and consolidation & & $13(30)$ & $11(39)$ \\
\hline
\end{tabular}

${ }^{\mathrm{a}}$ Values are expressed as No. (\%).

When we examine the CT findings according to chronic diseases; One patient with pleural effusion had a chronic renal failure (CRF), hypertension (HT), and diabetes mellitus (DM) and was older than 60 years. Pleural thickening was present in two patients. One of these patients was the $\mathrm{CRF}$, and the other was a patient with Behcet's disease (BD). Pericardial effusion was present in two female patients.
One patient had DM and HT, but the other patient had no chronic disease. These patients were over 60 years old. LAP was present in only one BD patient. We detected the halo sign in a patient with diabetes and hypertension. One of the two patients with fibrotic lines had DM and HT, and the other had BD. In the other two patients with the fibrotic line, there was no chronic disease. However, it was over 75 


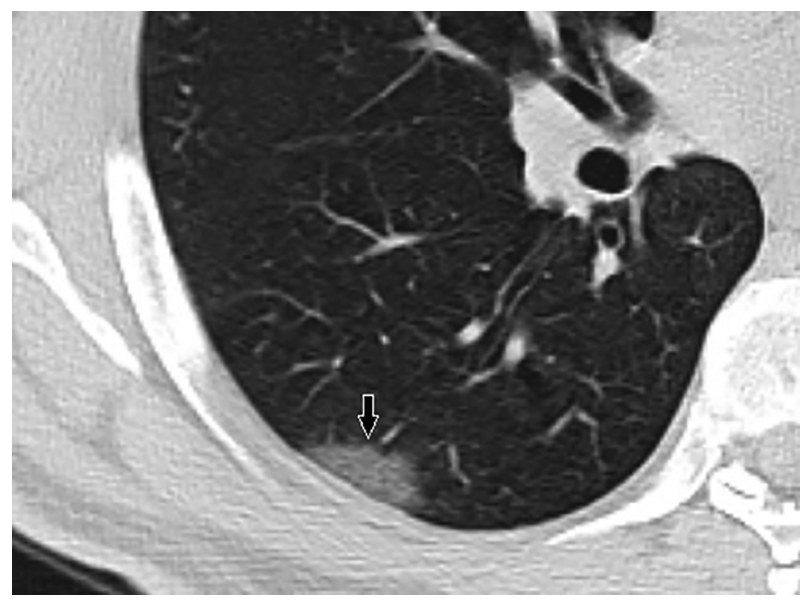

Figure 1. A39-year-old man with dry cough and fatigue. The black arrow on the axial $\mathrm{CT}$ of the right lung shows ground-glass opacity with the peripheral distribution.

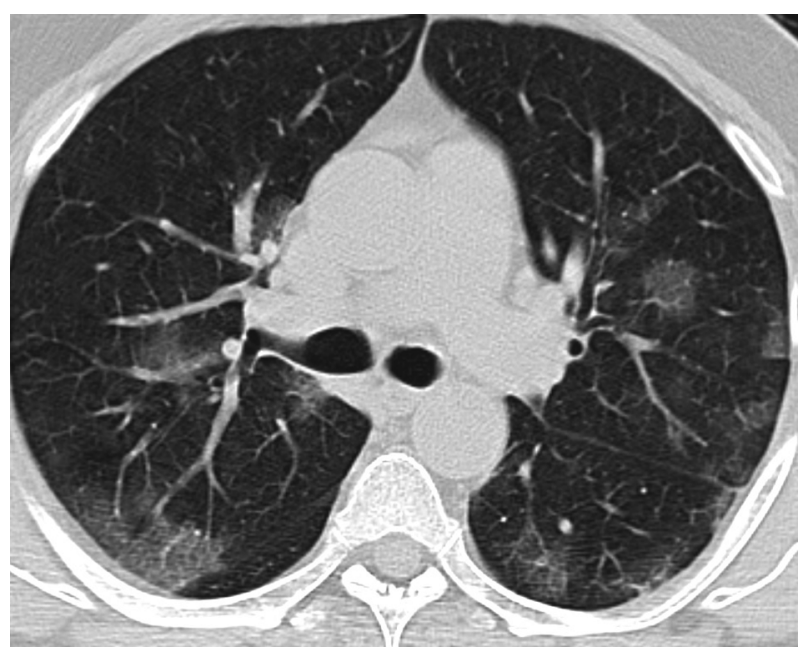

Figure 2. A 65-year-old man with fever and myalgia. Axial CT scan shows bilateral patch and round ground-glass opacities.

years old in four patients with the fibrotic line.

The mean age of patients with HT $(\mathrm{n}=20)$ was greater than patients without $\mathrm{HT}(65.4 \pm 9.0$ vs $56.5 \pm 12.9$ years, $\mathrm{P}=0.003)$. However, there was no difference between the two groups in terms of $\mathrm{CT}$ findings. When the age range of 40 - 60 was examined, two patients had HT, two patients had DM, two patients had DM, and HT, one patient, had Behcet's disease, and HT, one patient, had hypothyroidism and HT. Although only 6 (23.0\%) out of 26 patients in this age range had a chronic disease, 18 (67.0\%) cases had CT findings (highly suggestive for COVID-19 pneumonia). In this age group, four patients had CT findings for suspicious

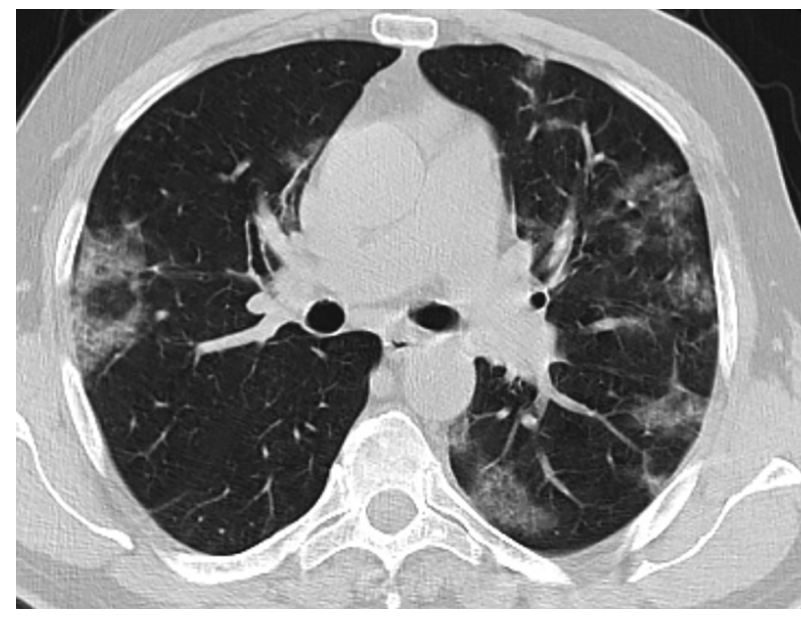

Figure 3. 43-year-old man with fever and dry cough. Axial CT image shows bilateral, multilobar, patchy ground-glass opacities.

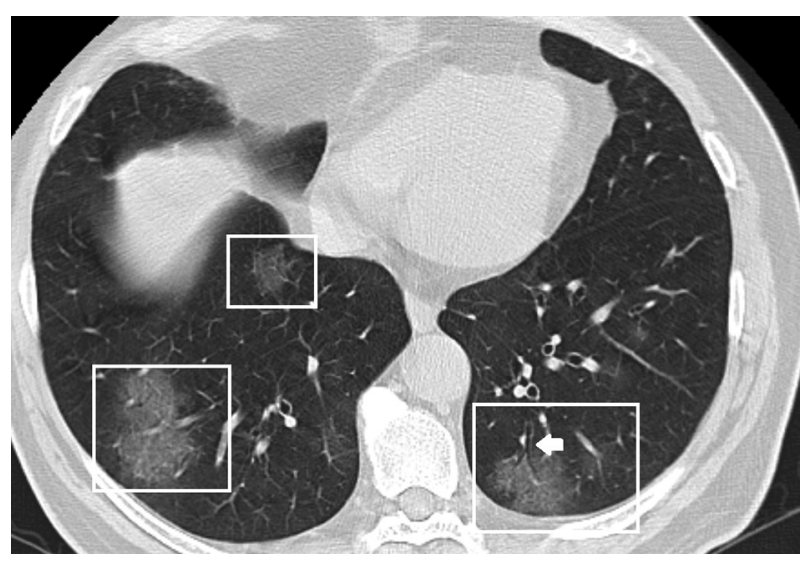

Figure 4. A 56-year-old man with fever and cough. Axial CT shows bilateral, multilobar, ground-glass opacities with air bronchogram (White arrow).

COVID-19 pneumonia despite the absence of chronic disease. In the group, 16 patients had COVID-19 CT findings (suspicious and highly suggestive); however, there were no chronic diseases. Interestingly, there were findings related to COVID-19 pneumonia in three patients who did not have any chronic diseases between the ages of 20 - 40 years.

\section{Discussion}

Today, COVID-19 is considered in all patients who admit to hospitals with the complaint of fever, cough, and dyspnea. It has been reported in many studies that the RT-PCR test has low sensitivity, but the $\mathrm{CT}$ examination is very sensitive for COVID-19 infection (5). Therefore, it is important 


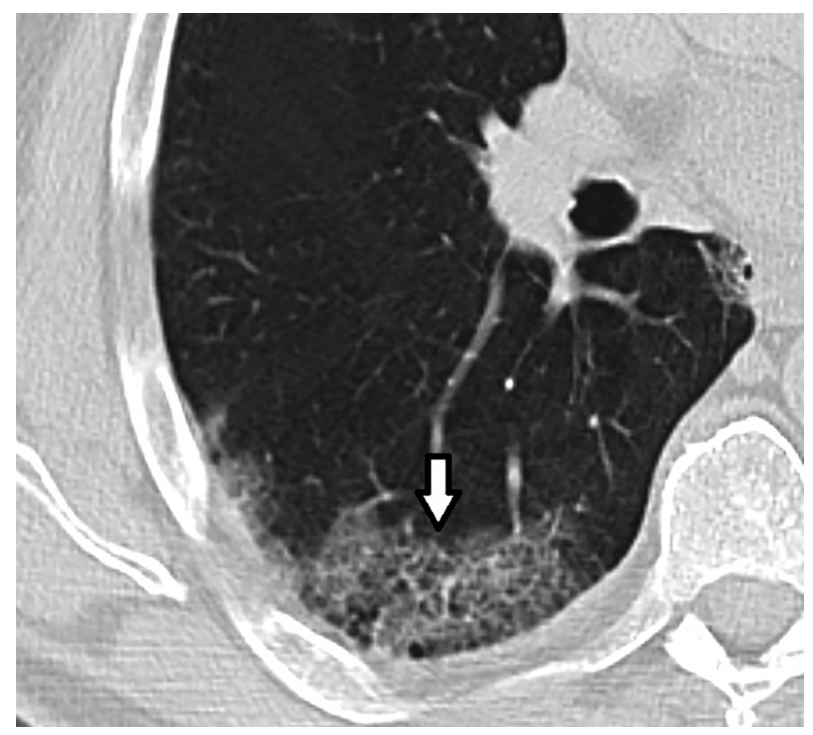

Figure 5. A 55-year-old woman with fever, cough, and myalgia. Axial CT of the right lung shows peripheral ground-glass opacity with a crazy-paving pattern (Arrow).

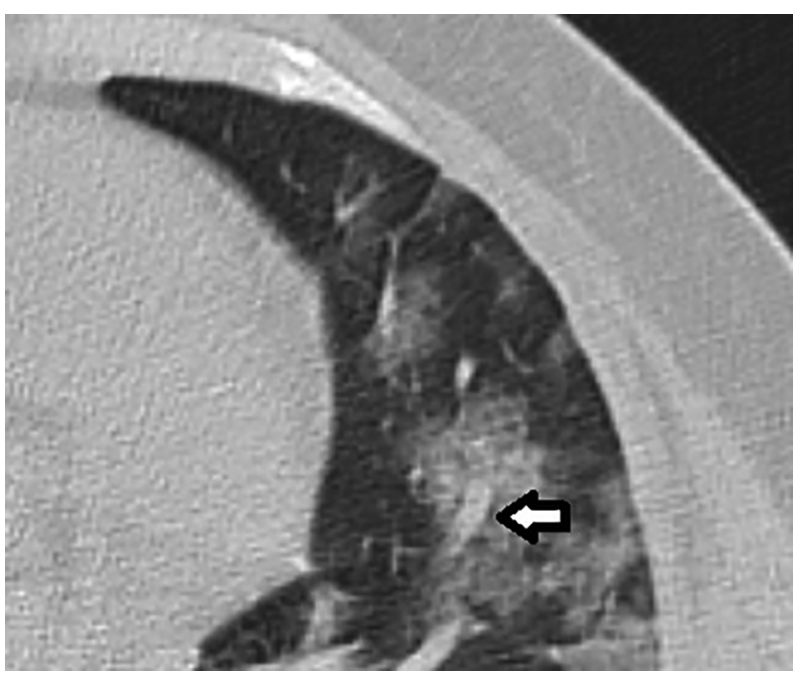

Figure 6. A 45-year-old man with a cough. Axial CT of left lung shows ground-glass opacity with vascular thickening (Arrow).

to define CT findings. Our results suggest that COVID-19 pneumonia is common in adults (mean age $=59$ ), as published before (7).

Nineteen of 63 patients (30\%), laboratory-confirmed with RT-PCR for COVID-19 pneumonia, had normal CT findings and classified as out of COVID-19 pneumonia. Patients diagnosed with COVID-19 by PCR were included in this study. We tested the CT findings of the patients diagnosed with COVID-19 with PCR. We thought initial CT should not

\begin{tabular}{lcc}
$\begin{array}{l}\text { Table 3. Specific and Other Findings in Initial Computed Tomography of Patients } \\
\text { with COVID-19 Pneumonia }{ }^{\mathrm{a}}\end{array}$ & $\begin{array}{c}\text { All Pneumonia } \\
\text { Groups }(\mathbf{N}=\mathbf{4 4})\end{array}$ & $\begin{array}{c}\text { Highly Suggestive } \\
\text { COVID-19 Pneumonia } \\
(\mathbf{N}=\mathbf{2 8})\end{array}$ \\
\hline Findings & $22(50)$ & $22(79)$ \\
\hline Crazy-paving pattern & $27(61)$ & $26(93)$ \\
\hline Air bronchogram & $17(39)$ & $17(61)$ \\
\hline Vascular thickening & $1(2)$ & $1(4)$ \\
\hline Halo sign & $1(2)$ & $1(4)$ \\
\hline Reverse halo sign & $4(9)$ & $1(4)$ \\
\hline Fibrotic lines & $1(2)$ & $1(4)$ \\
\hline $\begin{array}{l}\text { lymphadenopathy } \\
\text { LAP) }\end{array}$ & $1(2)$ & $2(7)$ \\
\hline Pleural effusion & $2(5)$ & $2(7)$ \\
\hline Pleural thickening & $2(5)$ & \\
\hline Pericardial effusion & & 0 \\
\hline
\end{tabular}

${ }^{\mathrm{a}}$ Values are expressed as No. (\%).

use as a screening method for asymptomatic patients and combined with RT-PCR tests in patients with typical clinical symptoms. Patients having negative PCR but thorax CT findings are frequently encountered during the COVID-19 pandemic. In these patients, the COVID-19 diagnosis can be confirmed by the 2 nd or 3rd PCR test. To avoid confusion, we tested patients diagnosed with PCR by COVID-19.

We classified patients into three groups, according to typical CT features of COVID-19 pneumonia. CT findings of 28 patients (44\%) were highly suggestive for COVID-19 pneumonia. Using this classification may speed up communication with clinicians and radiologists. CT findings of patients with COVID-19 are bilateral, multilobar GGO, mostly in the lower lobe and right middle lobe (8-10). Less

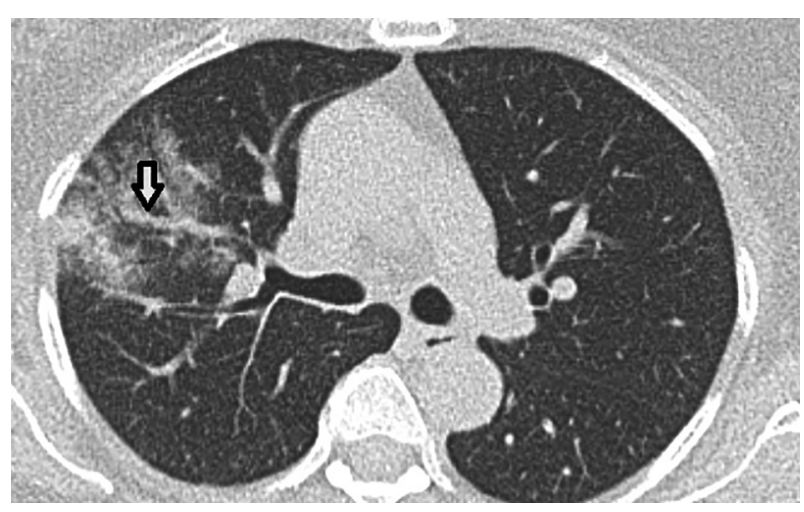

Figure 7. A 45-year-old man with fever, dry cough, and fatigue. Axial CT shows ground-glass opacity with vascular thickening (Arrow). 

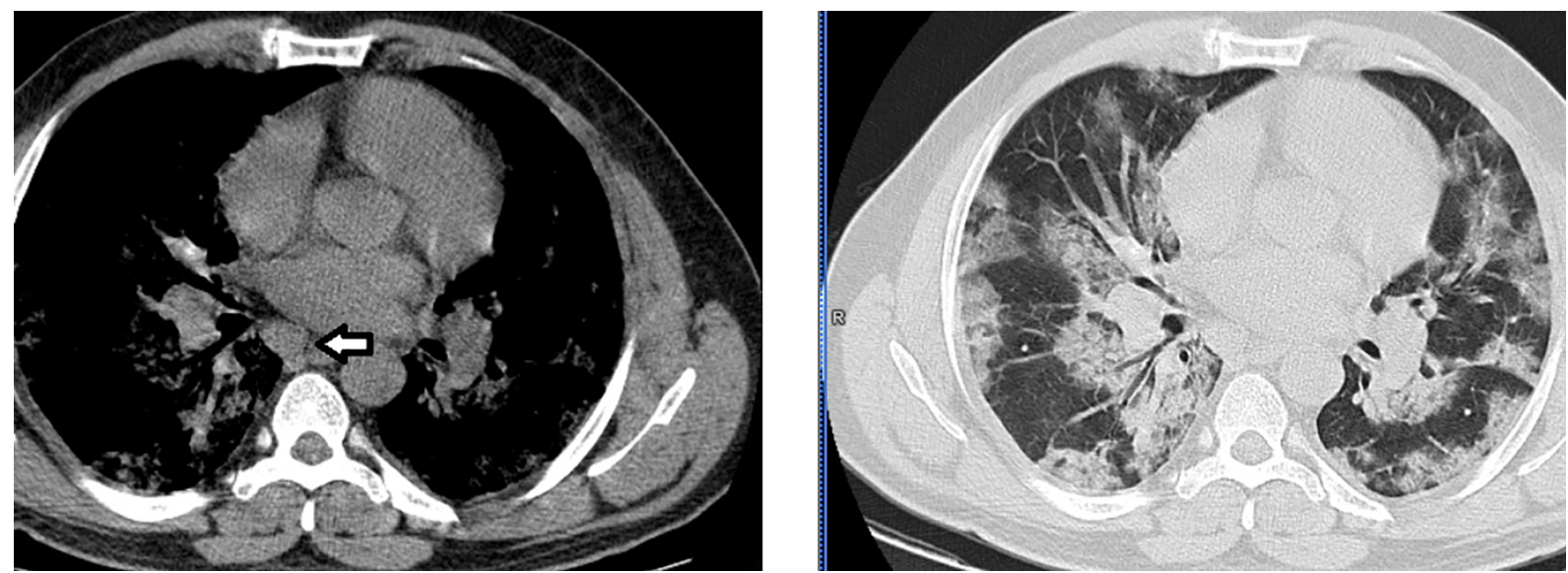

Figure 8. 46-year-old man with fever and cough. Axial CT scans in mediastinal (left) and lung (right) window settings show bilateral, multilobar patchy ground-glass opacities. Arrow shows lymphadenopathy, not a typical finding in Covid 19 pneumonia.

frequently, these findings may be GGO with a peripheral or posterior distribution (or both) (8-10). In our study, we found the most common CT findings of COVID-19 pneumonia as peripheral (64\%), multilobar (96\%), irregular and round (54\%) GGO (57\%). Air bronchogram (93\%), crazypaving patterns (79\%), and vascular thickening (61\%) were the most common special findings. Halo sign, reverse halo sign, LAP, pleural and pericardial effusion, pleural thickening, and fibrotic lines were rare findings. We detected these rare findings in patients with COVID-19 with chronic diseases such as DM, HT, BD, and CRF. The presence of chronic disease affects the prognosis of COVID-19 pneumonia, and may also affect CT findings. These rare findings were not present in patients with COVID-19 pneumonia without chronic disease. Only two people without a chronic disease had a fibrotic line, but these patients were very old. We thought these rare findings may be due to chronic disease rather than COVID-19 pneumonia. These rare findings have not been associated with COVID-19 pneumonia in the literature (11).

Interestingly, we found that patients with no chronic disease between the ages of 40 - 60 years might have COVID-19 pneumonia. Our patients under 40 old were very few, but half of them had signs of COVID-19 pneumonia. Our patients in the age group had no chronic illness or drug abuse. Although COVID-19 is seen with all ages, it is more severe in the elderly and those with chronic diseases (12). The presence of COVID-19 pneumonia in younger and patients without chronic disease complicates when and to whom radiological tests will be performed. We used low dose thorax CT in radiological shots. However, patients are still exposed to radiation. A general and comprehensive guideline on COVID-19 pneumonia CT findings has occurred. However, what is the role of CT in young patients with PCR negative but symptoms? Since the mortality rate of COVID-19 pneumonia is very low in young patients. Most of our patients under the age of 60 with COVID-19 pneumonia did not have a chronic disease. We followed up none of our patients in the intensive care unit. All of our patients recovered with no complications. In COVID-19 pneumonia, it should be specified whether younger patients' thorax CT should be evaluated.

We had some limitations in this study. Only the first CT findings of the patients were evaluated. Subsequent CT evaluation of patients is not included in the study. Imaging features of different periods of the disease can be determined using follow-up CTs. The number of young patients (under 40 years) is low. Since there is no chronic disease in young patients, the findings of COVID-19 pneumonia can be better determined. In this study, the sensitivity and specificity of CT or PCR for the diagnosis of COVID-19 pneumonia was not studied.

\subsection{Conclusions}

A part of patients referred to CT at the onset of the disease has normal CT findings. Early CT features in COVID-19 pneumonia are peripheral, multilobar, patchy, and round ground-glass opacities, often with air bronchogram, crazypaving patterns, and vascular thickening. Rare findings such as LAP, pleural effusion, pericardial effusion, pleural thickening, and fibrotic lines are seen in the presence of concomitant disease. 


\section{Footnotes}

Authors' Contribution: Study concept and design: CK, PDY, and SB. Analysis and interpretation of data: NP and PDY. Drafting of the manuscript: CK and PDY. Critical revision of the manuscript for important intellectual content: NP and HV. Statistical analysis: PDY, CK, and SB.

Conflict of Interests: There is no conflict of interest.

Ethical Approval: We obtained ethics approval for this study from the local Ethics Committee in April 2020.

Funding/Support: There is no funding or support regarding the publication of this study.

\section{References}

1. Zhu N, Zhang D, Wang W, Li X, Yang B, Song J, et al. A novel coronavirus from patients with pneumonia in China, 2019. $N$ Engl J Med. 2020;382(8):727-33. doi:10.1056/NEJMoa2001017. [PubMed: 31978945]. [PubMed Central: PMC7092803].

2. World Health Organization. Coronavirus disease 2019 (COVID-19) situation report-42. Geneva: World Health Organization; 2020.

3. Huang P, Liu T, Huang L, Liu H, Lei M, Xu W, et al. Use of chest CT in combination with negative RT-PCR Assay for the 2019 novel coronavirus but high clinical suspicion. Radiology. 2020;295(1):22-3. doi: 10.1148/radiol.2020200330. [PubMed: 32049600]. [PubMed Central: PMC7233360].

4. Ye Z, Zhang Y, Wang Y, Huang Z, Song B. Chest CT manifestations of new coronavirus disease 2019 (COVID-19): a pictorial review. Eur Radiol. 2020;30(8):4381-9. doi: 10.1007/s00330-020-06801-0. [PubMed: 32193638]. [PubMed Central: PMC7088323].
5. Fang Y, Zhang H, Xie J, Lin M, Ying L, Pang P, et al. Sensitivity of chest CT for COVID-19: Comparison to RT-PCR. Radiology. 2020;296(2):E1157. doi: 10.1148/radiol.2020200432. [PubMed: 32073353]. [PubMed Central: PMC7233365].

6. Simpson S, Kay FU, Abbara S, Bhalla S, Chung JH, Chung M, et al. Radiological Society of North America Expert Consensus Statement on reporting chest CT findings related to COVID-19. Endorsed by the Society of Thoracic Radiology, the American College of Radiology, and RSNA. Radiol Cardiothorac Imaging. 2020;2(2). e200152.

7. Chen N, Zhou M, Dong X, Qu J, Gong F, Han Y, et al. Epidemiological and clinical characteristics of 99 cases of 2019 novel coronavirus pneumonia in Wuhan, China: A descriptive study. Lancet. 2020;395(10223):507-13. doi: 10.1016/S0140-6736(20)30211-7. [PubMed: 32007143]. [PubMed Central: PMC7135076].

8. Salehi S, Abedi A, Balakrishnan S, Gholamrezanezhad A. Coronavirus disease 2019 (COVID-19): A systematic review of imaging findings in 919 patients. AJR Am J Roentgenol. 2020;215(1):87-93. doi: 10.2214/AJR.20.23034. [PubMed: 32174129].

9. Han R, Huang L, Jiang H, Dong J, Peng H, Zhang D. Early clinical and CT manifestations of coronavirus disease 2019 (COVID-19) pneumonia. AJR Am J Roentgenol. 2020:1-6. doi: 10.2214/AJR.20.22961. [PubMed: 32181672].

10. Hosseiny M, Kooraki S, Gholamrezanezhad A, Reddy S, Myers L. Radiology perspective of coronavirus disease 2019 (COVID-19): Lessons from severe acute respiratory syndrome and middle east respiratory syndrome. AJR Am J Roentgenol. 2020;214(5):1078-82. doi: 10.2214/AJR.20.22969. [PubMed: 32108495].

11. Akcay S, Ozlu T, Yilmaz A. Radiological approaches to COVID-19 pneumonia. TurkJ Med Sci. 2020;50(SI-1):604-10. doi:10.3906/sag-2004-160. [PubMed: 32299200]. [PubMed Central: PMC7195987].

12. Cure E, Cumhur Cure M. Comment on "Organ-protective effect of angiotensin-converting enzyme 2 and its effect on the prognosis of COVID-19". J Med Virol. 2020. doi: 10.1002/jmv.25848. [PubMed: 32266994]. [PubMed Central: PMC7262145]. 